\title{
HOMOTOPY-ABELIAN LIE GROUPS
}

\author{
BY S. ARAKI, I. M. JAMES AND EMERY THOMAS ${ }^{1}$
}

Communicated by Hans Samelson, May 12, 1960

A topological group $G$ is said to be homotopy-abelian if the commutator map of $G \times G$ into $G$ is nulhomotopic. Examples can be given $^{2}$ of non-compact Lie groups which are homotopy-abelian but not abelian. The purpose of this note is to prove

Theorem. A compact connected Lie group is homotopy-abelian only if it is abelian.

Corollary. If a Lie group is homotopy-abelian, then its maximal compact connected subgroup is abelian.

Our proof depends on the theory of [6]. Thus we consider the Samelson "commutator" product ${ }^{3}$ in the homotopy groups of $G$, which is trivial when $G$ is homotopy-abelian. The product of $\alpha \in \pi_{p}(G)$ with $\beta \in \pi_{q}(G)$ is denoted by $\langle\alpha, \beta\rangle \in \pi_{p+q}(G)$, where $p, q \geqq 1$. If $h$ is a homomorphism of $G$ into another topological group then

$$
h_{*}\langle\alpha, \beta\rangle=\left\langle h_{*} \alpha, h_{*} \beta\right\rangle,
$$

where $h_{*}$ denotes the induced homomorphism. Note that $h_{*}$ is an isomorphism if $h$ is a covering map and $p, q \geqq 2$. Hence if two topological groups have a common universal covering group then their higher homotopy groups are related by an isomorphism which is compatible with the Samelson product. Let $\sigma \pi_{q}(G)$, where $q \geqq 1$, denote the subset of $\pi_{2 q}(G)$ consisting of elements $\langle\beta, \beta\rangle$, where $\beta \in \pi_{q}(G)$. We assert the following

Lemma. Let $G$ be a compact connected simple non-abelian Lie group of dimension $n$ and rank $l$. Then $\sigma \pi_{q}(G) \neq 0$, where $q=2 n / l-3$.

The proof is by application of (2.2) of [6]. We distinguish between the classical and exceptional cases, beginning with the latter.

Let $G$ be one of the exceptional groups. Then $n / l=p$, an odd prime number, and $G$ has no $p$-torsion (see [3]). The $\bmod p$ cohomology of $G$ is an exterior algebra on a basis of $l$ generators. There is one generator $y$ in dimension $q$, while the remainder are of lower dimension. It follows from Proposition 6 on page 291 of [8] that $y$ has a nontrivial

\footnotetext{
1 Research supported in part by U.S. Air Force Contract AF 49(638)-79.

${ }^{2}$ Such as the 2-dimensional affine group (example suggested by H. Samelson).

${ }^{3}$ The theory of the Samelson product is given in [5], for example.
} 
image under the homomorphism induced by some map of $S^{q}$ into $G$. Thus $y$ has nonzero index, in the sense of [6], with regard to some element $\beta \in \pi_{q}(G)$. By Borel's theorem ${ }^{4}$ the $\bmod p$ cohomology of $B$, the classifying space of $G$, is a polynomial algebra on a basis of $l$ generators which correspond under transgression to those of the exterior algebra. The generator $x$ corresponding to $y$ has a nontrivial image under the homomorphism induced by some map of $S^{q+1}$ into $B$. In the polynomial algebra let $M$ denote the ideal generated by all the basis elements except $x$. If $z$ is such a generator then

$$
\operatorname{dim} z<\operatorname{dim} x=q+1=2(p-1),
$$

and so $\mathcal{P}^{s} z \in M$, where $\mathcal{P}^{s}(s \geqq 0)$ denotes the Steenrod operator. Hence $\rho^{s} M \subset M$, by the Cartan product formula. This proves that $\rho^{1} x \in M$, since by the Adem relation [1] we have

$$
\mathbb{P}^{p-2} \mathbb{P}^{1} x=(p-1) \mathbb{P}^{p-1} x=(p-1) x^{p} \notin M .
$$

Hence $\mathcal{P}^{1} x \equiv c x^{2}, \bmod M$, where $c \neq 0$, and so $\mathcal{P}^{1} x$ is significant with regard to $\beta$, in the sense of [6]. Therefore $\langle\beta, \beta\rangle \neq 0$, by (2.2) of [6], which proves the lemma when $G$ is exceptional.

If $G$ is not exceptional then $G$ is locally-isomorphic to one of the classical groups:

$$
S U(l+1), \quad S O(2 l+1), \quad S p(l), \quad S O(2 l) .
$$

It is shown in $\S 4$ of [6] that each of

$$
\sigma \pi_{2 l+1} U(l+1), \quad \sigma \pi_{4 l-1} S O(2 l+1), \quad \sigma \pi_{4 l-1} S p(l),
$$

contains elements of odd order, and it follows from (18.2) of [4] that the same is true of $\sigma \pi_{4 l-5} S O(2 l)(l \neq 1)$. Furthermore

$$
\pi_{r} S U(l+1) \approx \pi_{r} U(l+1),
$$

under the injection, and so $\sigma \pi_{2 l+1} S U(l+1) \neq 0$. Since the Samelson product is an invariant of the structure class this completes the proof of the lemma.

To deduce the theorem we recall that a compact connected Lie group $G$ is locally isomorphic to $G^{\prime}$, say, where $G^{\prime}$ is the direct product of an abelian group $T$ with various nonabelian simple groups. When any of these latter are present there exists, by the lemma, some value of $q$ such that $\sigma \pi_{q}\left(G^{\prime}\right) \neq 0$ and hence $\sigma \pi_{q}(G) \neq 0$. Thus $G^{\prime}=T$ if $G$ is homotopy-abelian, and hence the theorem follows at once. A maximal

${ }^{4}$ See (7.2) and (19.1) of [2]. 
compact connected subgroup of a Lie group is a deformation retract of the component of the identity [7], and so the corollary is an immediate consequence of the theorem.

\section{REFERENCES}

1. J. Adem, Relations on iterated reduced powers, Proc. Nat. Acad. Sci. U.S.A. vol. 39 (1953) pp. 636-638.

2. A. Borel, Sur la cohomologie des espaces fibrês ..., Ann. of Math. vol. 57 (1953) pp. 115-207.

3. - Topology of Lie groups and characteristic classes, Bull. Amer. Math. Soc. vol. 61 (1955) pp. 397-432.

4. A. Borel and J.-P. Serre, Groupes de Lie et puissances reduites de Steenrod, Amer. J. Math. vol. 75 (1953) pp. 409-448.

5. I. M. James, On H-spaces and their homotopy groups, (to be published in Oxford Quart. J. of Math.).

6. I. M. James and E. Thomas, Which Lie groups are homotopy-abelian? Proc. Nat. Acad. Sci. U.S.A. vol. 45 (1959) pp. 737-740.

7. H. Samelson, Topology of Lie groups, Bull. Amer. Math. Soc. vol. 58 (1952) pp. 2-37.

8. J.-P. Serre, Groupes d'homotopie et classes de groupes abeliens, Ann. of Math. vol. 58 (1953) pp. 258-294.

INSTITUTE FOR ADVANCED STUdy, OXFORD UNIVERSITY AND

UNIVERSITY OF CALIFORNIA AT BERKELEY 